\title{
Continuous occurrence of intra-individual chromosome rearrangements in the peach potato aphid, Myzus persicae (Sulzer) (Hemiptera: Aphididae)
}

\author{
Valentina Monti • Giorgia Lombardo • \\ Hugh D. Loxdale • Gian Carlo Manicardi • \\ Mauro Mandrioli
}

Received: 10 February 2012/Accepted: 21 May 2012/Published online: 27 May 2012

(C) Springer Science+Business Media B.V. 2012

\begin{abstract}
Analysis of the holocentric mitotic chromosomes of the peach-potato aphid, Myzus persicae (Sulzer), from clones labelled 50, 51 and 70 revealed different chromosome numbers, ranging from 12 to 14 , even within each embryo, in contrast to the standard karyotype of this species $(2 \mathrm{n}=12)$. Chromosome length measurements, combined with fluorescent in situ hybridization experiments, showed that the observed chromosomal mosaicisms are due to recurrent fragmentations of chromosomes X, 1 and 3. Contrary to what has generally been reported in the literature, $\mathrm{X}$ chromosomes were frequently involved in recurrent fragmentations, in particular at their telomeric ends opposite to the nucleolar organizer region. Supernumerary B chromosomes have been also observed in clones 50 and 51. The three aphid clones showed recurrent fissions of the same chromosomes in the same regions, thereby suggesting that the $M$. persicae genome has fragile sites that are at the basis of the observed changes in chromosome number. Experiments to induce males also revealed that $M$. persicae clones 50, 51 and 70 are obligately parthenogenetic, arguing that the reproduction by apomictic parthenogenesis favoured the stabilization and inheritance of the observed chromosomal fragments.
\end{abstract}

V. Monti · G. Lombardo · M. Mandrioli ( $\square)$

Dipartimento di Biologia, Università di Modena e Reggio

Emilia, Via Campi 213/D, 41125 Modena, Italy

e-mail: mauro.mandrioli@unimo.it

V. Monti · G. C. Manicardi

Dipartimento di Scienze Agrarie e degli Alimenti, Università di

Modena e Reggio Emilia, Reggio Emilia, Italy

H. D. Loxdale

Royal Entomological Society, The Mansion House, Chiswell

Green Lane, St. Albans, UK
Keywords Aphids - Myzus persicae $\cdot$ Holocentric chromosomes - Satellite DNAs - Fragile sites - Karyotype rearrangements $\cdot$ Parthenogenesis

\section{Introduction}

Aphids (Hemiptera: Aphididae) are ancient insects (indeed fossils go back to the Triassic, about 220-210 Myr) (Grimaldi and Engel 2005) that have conquered most of the world's biomes, including the tropics and subarctic regions (Loxdale 2009), whilst they infest a huge range of plants, usually monophagously, although some species appear to be polyphagous (Loxdale 2009).

Aphids reproduce primarily by apomictic parthenogenesis, a form of reproduction whereby adult females give birth to female progeny in the absence of male fertilization (Soumalainen et al. 1987). It has been frequently suggested that no genetic recombination occurs in such parthenogenetic generations (Blackman 1985; Soumalainen et al. 1987); hence, it has been assumed that the offspring represent a 'genetically identical' clone (Dixon 1989). In view of this assumed genetic identity, Janzen (1977) referred to aphid species as a 'superorganism' or as a single 'evolutionary individual, able to exploit a much larger geographic region and its resources (Loxdale 2008a).

Actually, aphid lineages within the same species can differ in colour and size (Loxdale 2008b), intrinsic rate of increase (Jenkins 1991), ovariole number (Dixon 1989), reproductive modes (Loxdale 2008b), ability to transfer pathogenic plant viruses (Terradot et al. 1999), and susceptibility/resistance to predators, parasites, pathogens and pesticides (Losey et al. 1997; Devonshire et al. 1999; Loxdale 2008b). This strongly suggests that clonality in aphids has been overestimated, thereby making the 
evaluation of the true nature and reality of the clone intriguing both empirically and conceptually (Loxdale 2008a, b, 2009).

In the last 15 years, a growing number of studies has demonstrated that aphid asexual lineages are not true clones, since their genome can rapidly mutate (as expected since DNA is a very dynamic, rapidly mutating molecule; Loxdale 2010a), and that these variations are selectable and may affect some phenotypic traits, such as plant host choice (Loxdale 2008a, b, 2009). As a result, the true nature of the aphid clone, and indeed clones in general, is not simply just one of semantics, but rather, concerns a scientifically important re-appraisal, perhaps including re-definition of the phenomenon (Martens et al. 2009).

Molecular analyses, such as random amplified polymorphic DNA (RAPDs) and amplified fragment length polymorphism (AFLP), have provided unequivocal evidence of mutational changes within aphid lineages, most of which are due to somatic events, mainly affecting noncoding regions, although some are in the germ line (e.g. De Barro et al. 1995; Lushai et al. 1998; Loxdale and Lushai 2007; Vorwerk and Forneck 2007; Loxdale 2008a, b, 2010b). Similarly, intraclonal, intermorph RAPD-based mutations have also been reported in clones of the grain aphid, Sitobion avenae (F.) (Lushai et al. 1997), whilst both Fenton et al. (1998) and Shufran et al. (2003) have also revealed variations in the ribosomal intergenic spacer (IGS) region of aphids. Lastly, Mandrioli et al. (1999a) reported somatic recombination events between the homologous nucleolar organizing regions (NORs) located on the X chromosome supporting the presence of recombinational events in aphid clonal lineages.

The presence of genetic differences among clones is potentially very important in worldwide attempts to control aphid pests. This is because several studies have clearly shown that cryptic sympatric speciation occurs in a wide range of aphid species (Loxdale and Lushai 2007; Loxdale 2008a, 2010b), including evidence of rapid chromosomal changes affecting host adaptation-speciation events, as, for example, in the corn leaf aphid, Rhopalosiphum maidis (Fitch) and in the global insecticide resistant pest, the peach potato aphid, Myzus persicae (Sulzer) (Blackman 1987; Brown and Blackman 1988; Field and Blackman 2003).

In view of their impact in agriculture (in particular, in terms of plant pathogenic virus transmission), aphids need to be controlled by pesticides and/or using biological control agents (see Van Emden and Harrington 2007). However, in the absence of a thorough understating of the genetics of aphid populations/clones, it is difficult to properly evaluate the presence of transmissible and adaptive variations that may well make biological and chemical controls less effective (Loxdale 2008b; Foster et al. 2007;
Fenton et al. 2010; van Toor et al. 2012, in press). In light of this, in the present paper, we have investigated three clones of Myzus persicae, analyzing their karyotypes in detail using two chromosomal markers (an X-chromosome specific Hind200 satellite and a subtelomeric repeat). We provide evidence of cytogenetic changes at both at the inter- and intra-clonal level, highlighting the extremely dynamic nature of the aphid clone, even at the level of karyotype.

\section{Materials and methods}

Specimens of $M$. persicae were obtained from the following asexual lineages (=clones): 1 , collected in Pisa (Central Italy) on peach plants; $33 \mathrm{H}$, a laboratory strain derived from the US1L strain kindly provided by Alan Devonshire (Rothamsted Research, Harpenden, UK); 50 and 51, both collected in Salerno (South Italy) on tobacco plants; and 70 , collected in Cosenza (South Italy) on peach plants. Each population was established as a clone from a single female aphid originally collected from the field and thereafter maintained as a colony of parthenogenetic females on pea (Pisum sativum) plants at $19{ }^{\circ} \mathrm{C}$ with a light-dark regime of $16 \mathrm{~h}$ light and $8 \mathrm{~h}$ darkness.

Males were induced by exposing parthenogenetic females to short photoperiods ( $8 \mathrm{~h}$ light: $16 \mathrm{~h}$ dark), according to Crema (1979) at $18{ }^{\circ} \mathrm{C}$.

Chromosome preparations were made from parthenogenetic females by spreading embryo cells and by squash preparation of single embryos as reported by Mandrioli et al. (1999b). C banding treatment was performed according to the technique of Sumner (1972) and was followed by staining with DAPI, according to Donlon and Magenis (1983), and chromomycin $\mathrm{A}_{3}\left(\mathrm{CMA}_{3}\right)$, as described in Schweizer (1976).

DNA extraction, following a standard phenol-chloroform protocol, whilst dot-blotting experiments were performed as described in Mandrioli et al. (1999b).

PCR digoxigenin labelling of the subtelomeric repeat was performed with a PCR DIG labelling kit according to the Roche protocol using the specific oligonucleotide primers MpR-F (5'-TCAAAGTTCTCGTTCTCC- $\left.3^{\prime}\right)$ and MpR-R (5'-GTTTTAACAGAGTGCTGG-3'), designed according the subtelomeric repeat sequence available in the literature (Spence et al. 1998). The reaction conditions were $94{ }^{\circ} \mathrm{C}$ for $90 \mathrm{~s}$ (denaturation), a total of 25 cycles of $94{ }^{\circ} \mathrm{C}$ for $30 \mathrm{~s}, 51{ }^{\circ} \mathrm{C}$ for $30 \mathrm{~s}$ (annealing) and $72{ }^{\circ} \mathrm{C}$ for 30 $\mathrm{s}$ (extension), and with a final extension step at $72{ }^{\circ} \mathrm{C}$ for 7 min.

The Hind200 probe was isolated by digesting M. persicae genomic DNA with the restriction enzyme HindIII at $37{ }^{\circ} \mathrm{C}$ for $16 \mathrm{~h}$ and eluting from the $1.2 \%$ agarose gel the 
band corresponding to the Hind200 satellite monomers (Genbank AF161255), according to Mandrioli et al. (1999b). The eluted DNA was labelled using a random priming DIG labelling kit according to the Roche Diagnostics (Mannheim, Germany) protocol.

Fluorescent in situ hybridization (FISH) was performed as described by Mandrioli et al. (2011). FISH slides were observed using a Zeiss Axioplan epifluorescence microscope. Photographs of the fluorescent images were taken using a CCD camera (Spot, Digital Instrument, Madison, USA) and the Spot software supplied with the camera and processed using Adobe Photoshop (Adobe Systems, Mountain View, CA).

For each different karyotype observed in the three clonal populations, measurements of chromosome length were performed on 50 metaphases using the software MicroMeasure, available at the Biology Department at Colorado State University website (http://rydberg.biology.colostate. edu/MicroMeasure).

\section{Results}

The standard karyotype of the aphid $M$. persicae, as assessed by specimens of clone 1, comprised 12 chromosomes, including five pairs of autosomes and two X chromosomes that can be easily identified since they are the longest in the complement (Fig. 1).

The cytogenetic study of the M. persicae clones 50, 51 and 70 revealed, after propidium iodide staining, that these three clones showed a strong mosaicism, both at the intraindividual and inter-individual level. In particular, $80 \%$ of the metaphase plates observed in clone 50 consisted of 13 chromosomes whilst just $20 \%$ showed the usual karyotype of 12 chromosomes. Analysis of clone 70 showed that $70 \%$ of the observed metaphases had 14 chromosomes, $21.5 \%$ of which possessed 13 chromosomes, whilst $8.5 \%$ had the standard karyotype of $2 \mathrm{n}=12$. Lastly, clone 51 showed $78.5 \%$ of the observed plates to have 13 chromosomes and $21.5 \%$ with the typical karyotype of 12 chromosomes.

With the aim of identifying the chromosomes involved in the observed karyotype changes, we combined FISH experiments, performed with a subtelomeric satellite DNA that labelled all the $M$. persicae chromosome ends with the exception of the rDNA telomere in each $\mathrm{X}$ chromosome (Spence et al. 1998), with the micro-measurements of each chromosome (Figs. 1, 2, 3, 4). This approach, previously successfully used by Monti et al. (2012), revealed that the mosaicism observed in clones 50, 51 and 70 is due to chromosomal fragmentations, even if it was not possible to clearly identify (at least in some clones) the actual chromosomes involved in the various observed karyotypic changes. In a standard karyotype stained using FISH to show the subtelomeric satellite DNA, five pairs of autosomes were observed with both the telomeres labelled with the subtelomeric probes and two $\mathrm{X}$ chromosomes with a single labelled end (Fig. 1).

Specimens of $M$. persicae strain 50 showed metaphases with 12 and 13 chromosomes. Interestingly, karyotypes with 13 chromosomes were due to the presence of a supernumerary chromosome with both the ends labelled after FISH (Fig. 2). Similar to clone 50, metaphase plates of aphids of clones 51 showed either 12 or 13 chromosomes. However, $50 \%$ of the plates with 13 chromosomes had a fission to have occurred at one $\mathrm{X}$ chromosome, of which $25 \%$ was due to fission of autosome 3 and $25 \%$ to the presence of a small chromosome with both the termini labelled after FISH
Fig. 1 FISH with the FITClabeled subtelomeric probe, combined with the micromeasure analysis of the chromosome length, allowed the identification of each twelve $M$. persicae chromosome (stained with propidium iodide) from clone 1 (a) and the construction of a corresponding karyogram (b). Bar corresponds to $10 \mu \mathrm{m}$

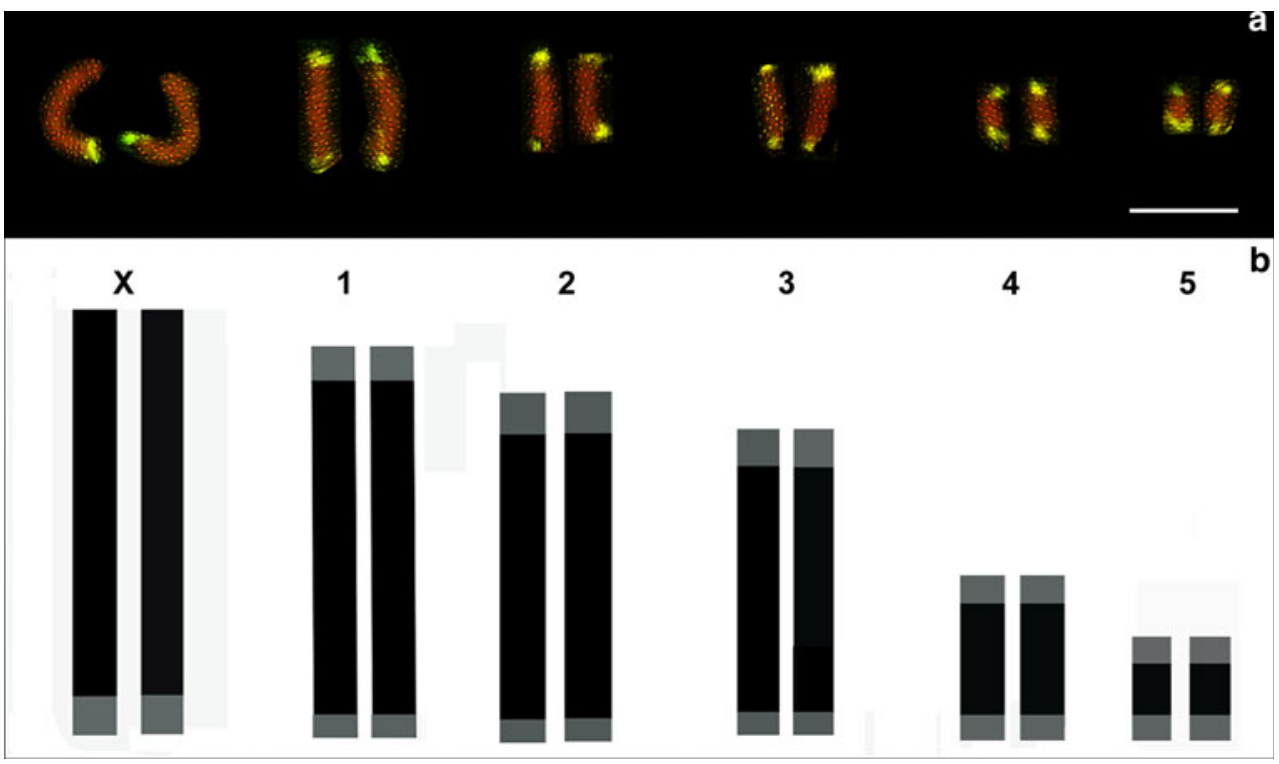




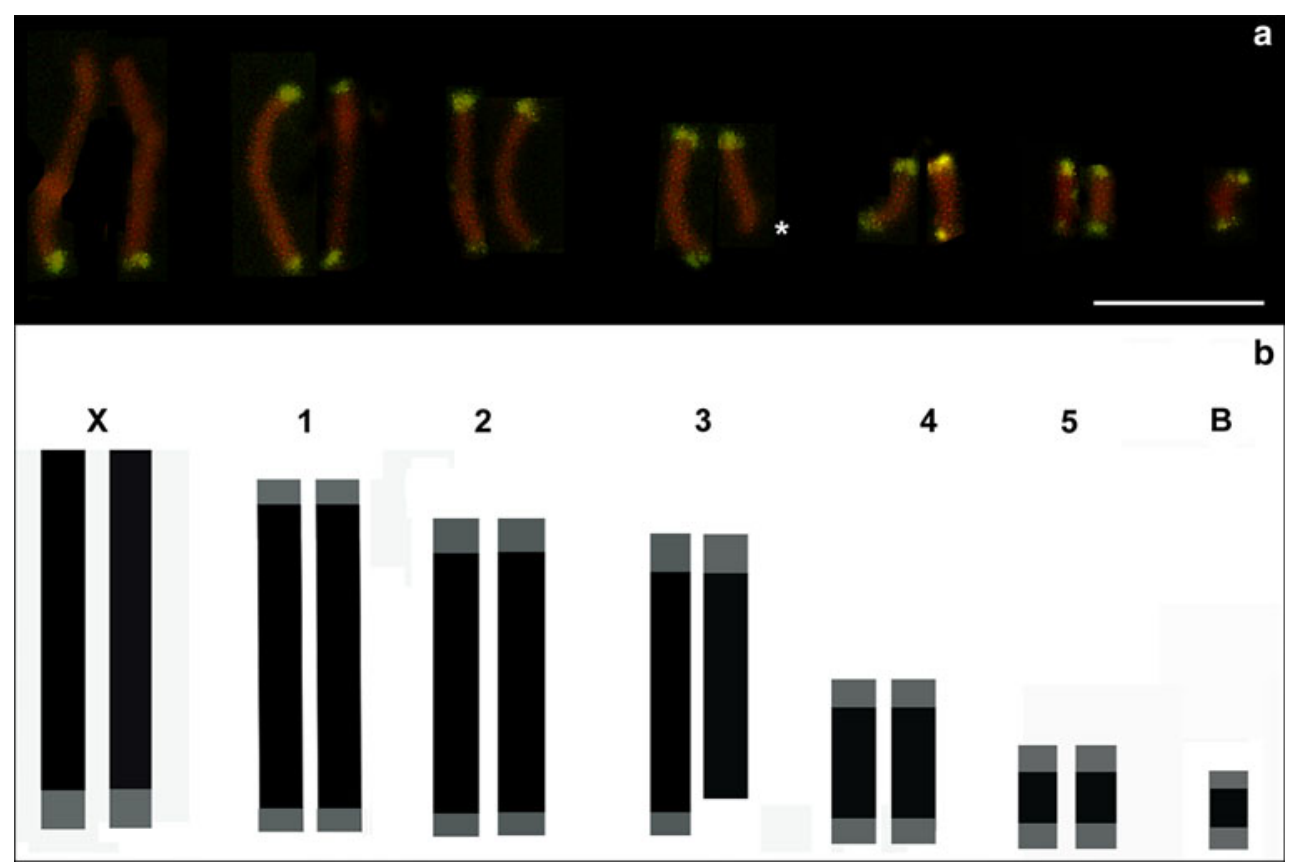

Fig. 2 As showed by FISH with the FITC-labeled subtelomeric probe, $M$. persicae clone 50 presented unusual plates with 13 chromosomes (stained with propidium iodide) due to the presence of

with the subtelomeric probe (Fig. 3a-c). Unexpectedly, plates with fissions occurring at the $\mathrm{X}$ chromosome also showed a deletion of a subtelomeric region on autosome 3 (Fig. 3a-c).

Analysis of the deleted X chromosome after hybridization with the subtelomeric probe suggested that the deletion probably occurred at the NOR bearing telomere and we therefore performed $\mathrm{CMA}_{3}$ and silver staining to confirm this hypothesis (Fig. 3d, e). Both staining approaches showed that the $\mathrm{X}$ fragment did not contain the ribosomal DNA genes so that the observed fission of the $\mathrm{X}$ chromosome involved an interstitial region rather than the telomeric one (Fig. 3f). Furthermore, the presence of heteromorphism in terms of the size of the $\mathrm{CMA}_{3}$ stained-telomeres revealed that the fission always occurred in the $\mathrm{X}$ chromosome possessing the smaller $\mathrm{CMA}_{3}$-positive telomere.

Specimens of clone 70 showed metaphases with chromosome number ranging from 12 to 14 . In about $70 \%$ of karyotypes comprising 13 chromosomes, fission was due to a single break involving the $\mathrm{X}$ chromosome (Figs. 4a), whereas $30 \%$ of the observed plates showed fissions occurring at autosome 3 (Fig. 4b). Metaphase plates comprising 14 chromosomes resulted from simultaneous fragmentations occurring on chromosomes 1 and 3 (25\% of the observed varitypes) (Fig. 4c), or alternatively because of fissions involving one $\mathrm{X}$ chromosome and one autosome 3 (75\% of the observed varitypes) (Fig. 4d). We never observed plates with 13 chromosomes involving autosome a supernumerary chromosome, together with a deleted autosome 3 (indicated by the asterisk) (a) as evident in the corresponding karyogram (b). Bar corresponds to $10 \mu \mathrm{m}$

1 alone, suggesting that autosome 1 was not frequently involved in such fissions.

In order to confirm the presence of B chromosomes in M. persicae clones 50 and 51, a DAPI staining of $\mathrm{C}$ banded chromosomes was performed, thereby revealing that in each clone, a small supernumerary chromosome exists, highly stained by DAPI and enriched in heterochromatin (Fig. 5).

The comparison of the hybridization pattern of the subtelomeric satellite suggested a different amplification of this repeated DNA in the analyzed clones. After FISH experiments, some clones such as clone 70 (Fig. 6a) presented larger subtelomeric areas than others, as evident for clone 51 (Fig. 6b). Dot-blot experiments with the subtelomeric probe confirmed this difference and showed that the amount of subtelomeric satellite DNA was greater in clones 50 and 70 than in clones 1 (used as control with standard karyotype) and 51, respectively, revealing the occurrence of clone-specific amplification of the subtelomeric regions of the chromosomes.

Differences among clones were not limited to the presence of variant karyotypes, since rearrangements were also observed upon comparing plates with the standard $2 \mathrm{n}=12$ chromosome number. Indeed, applying the FISH approach using the Hind 200 probe we showed that the X chromosomes could have a different hybridization pattern (Fig. 7). In particular, clones $33 \mathrm{H}$ (previously analysed by Monti et al. 2012) (Fig. 7a), 51 (Fig. 7c), 70 (Fig. 7d) and 1 (Fig. 7e) showed four bands on the $\mathrm{X}$ chromosomes after 

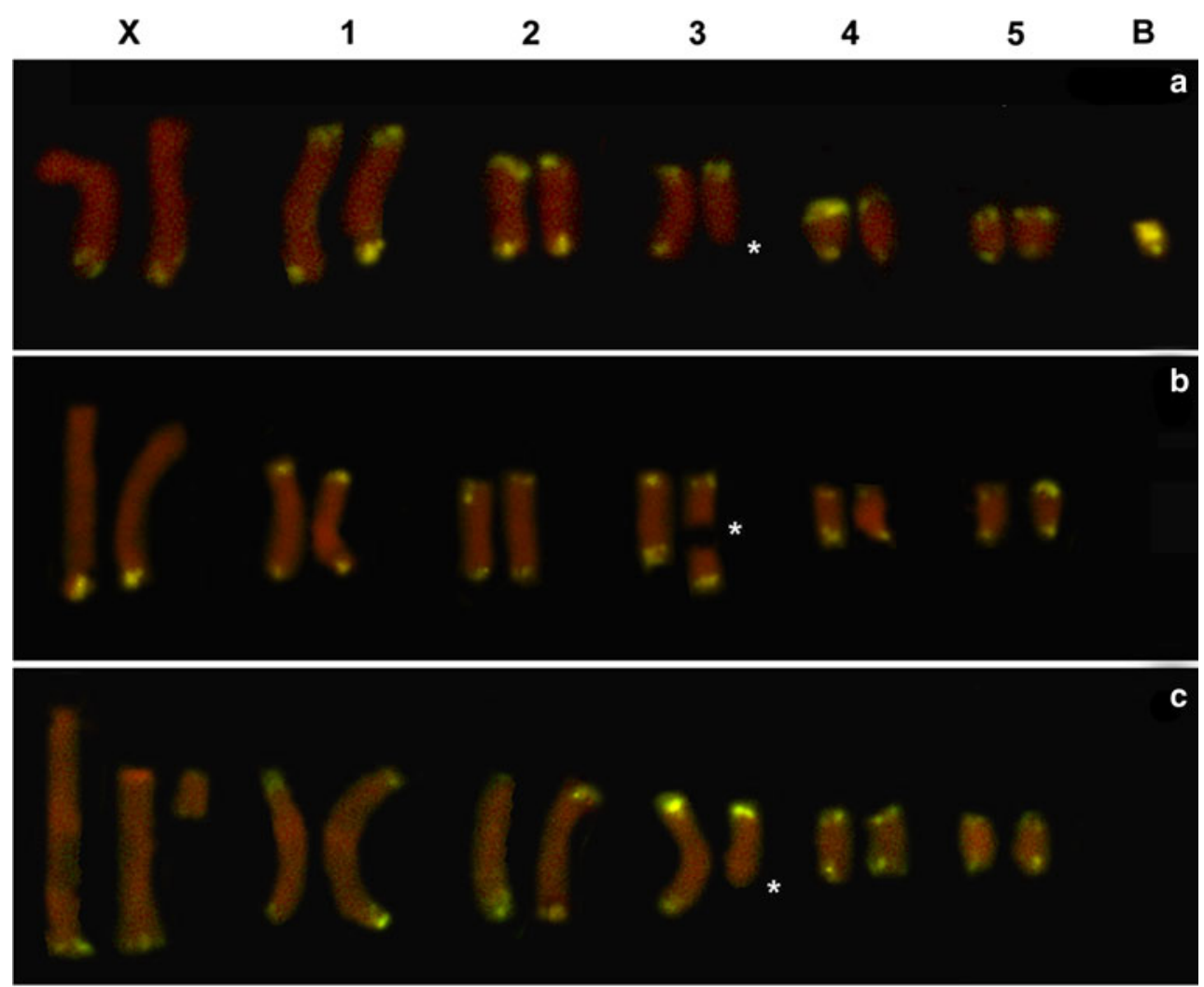

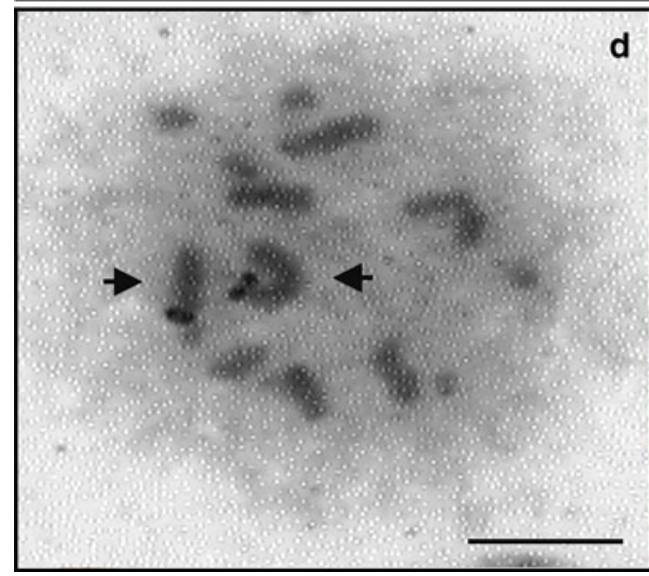

d

Fig. 3 FISH with the FITC-labeled subtelomeric probe on propidium iodide-stained chromosomes showed three different $2 \mathrm{n}=13$ karyotype variants in $M$. persicae clone 51 consisting of plates with a small supernumerary chromosome with both the termini labelled after FISH with the subtelomeric probe (a), metaphases with a fission occurred at one autosome 3 (indicated by the asterisk) (b) and plates with a

Hind200 hybridization, whereas clone 50 (Fig. 7b) showed five bands, suggesting that a duplication of a Hind200 band occurred. Interestingly, clones 1 and $33 \mathrm{H}$ presented a band in a different position on the $\mathrm{X}$ chromosomes than clones 51 and 70, clearly indicating that an inversion event had occurred (Fig. 7f).

Attempts at male induction revealed that $M$. persicae clones 50,51 and 70 were anholocyclic, since it was not possible to produce the sexual morphs, contrary to the

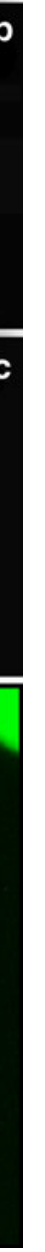

fission of one $\mathrm{X}$ chromosome (c). Silver (d) and $\mathrm{CMA}_{3}$ staining (e) indicate the fission at the $\mathrm{X}$ chromosome did not involved the NOR bearing telomere of the $X$ chromosomes but it is due to a deletion of an intercalary portion of the $\mathrm{X}$ chromosome, as showed in the corresponding karyogram (f). Arrows indicate the $\mathrm{X}$ chromosomes. Bar corresponds to $10 \mu \mathrm{m}$

situation obtained under the same experimental conditions with the holocyclic $M$. persicae clone 1 used as a control, and previously reported in Monti et al. (2012).

\section{Discussion}

Recent use of molecular and cytogenetic markers suggests that the genome of aphids belonging to asexual lineages 

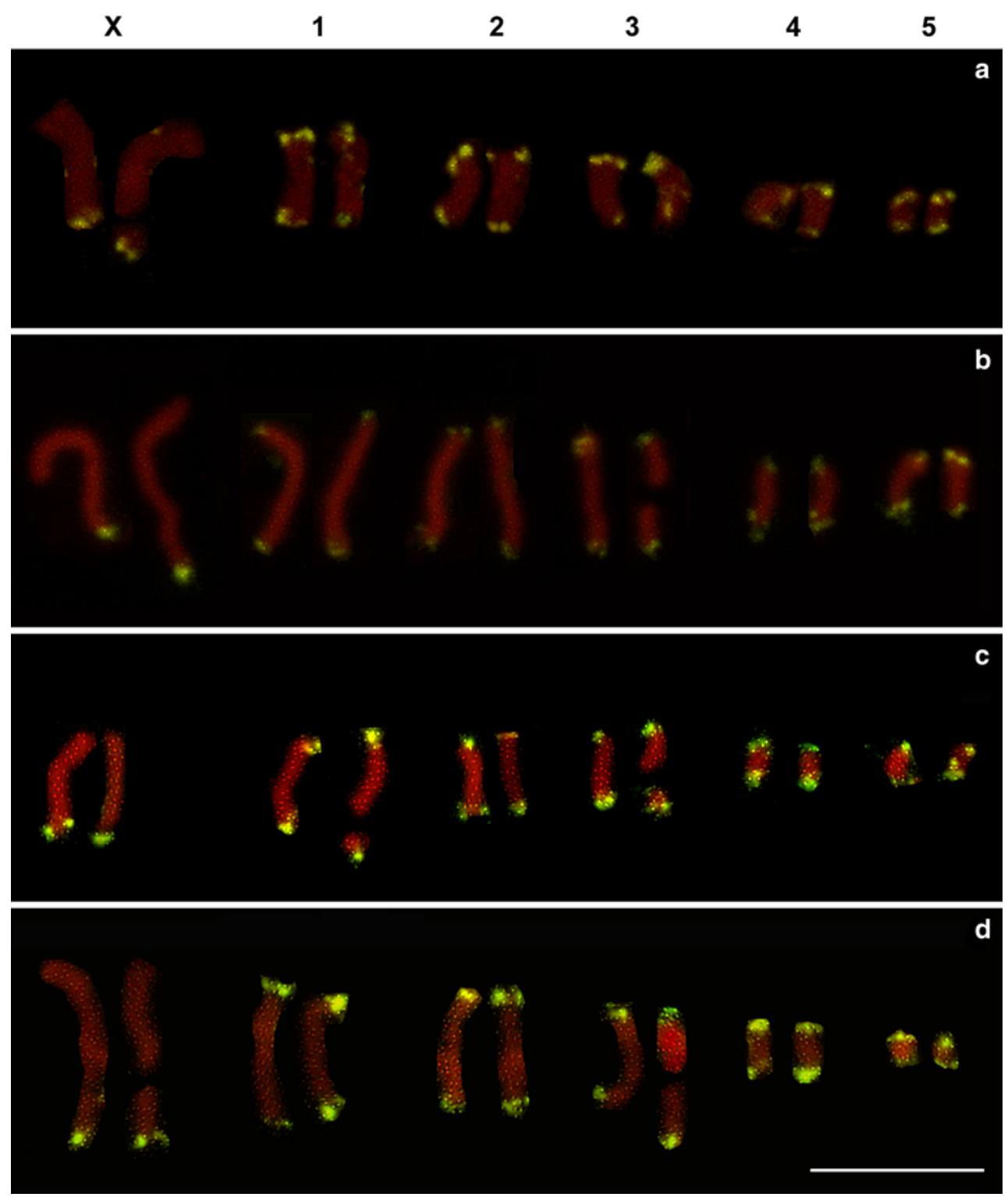

Fig. 4 As showed by FISH with the FITC-labeled subtelomeric probe, $M$. persicae clone 70 presented plates with chromosome number ranging from 12 to 14 . Karyotypes consisting of 13 chromosomes were due to a single fission involving a single $\mathrm{X}$ chromosome (a) or a fission occurring at autosome 3 (b). Metaphase

rapidly accumulate different kind of mutations (Loxdale 2008a, b, 2009, 2010b). In this respect, the presence of chromosome polymorphisms can be extensive in aphids, as reported in Trama maritime (Eastop), where specimens of the same species had different karyotypes (inter-individual polymorphisms) (Blackman et al. 2000), and in the $M$. persicae clone $33 \mathrm{H}$, where an extensive intraclonal chromosomal mosaicism was observed (Monti et al. 2012). Furthermore, differences due to recombination of the ribosomal DNA genes between the two $\mathrm{X}$ chromosomes were observed in diverse aphid species, showing that plates consisting of 14 chromosomes resulted from simultaneous fragmentations of chromosomes 1 and 3 (c) or of fissions involving one $\mathrm{X}$ chromosome and one autosome 3 (d). Chromosomes have been counterstained with propidium iodide. Bar corresponds to $10 \mu \mathrm{m}$

karyotypic changes can occur rapidly within aphid populations (Mandrioli et al. 1999a, c).

Until now, studies of different $M$. persicae clones have clearly demonstrated that this species not only comprises different populations with standard $2 \mathrm{n}=12$ karyotypes, but also several populations bearing different types of genome recombination and/or variant karyotypes (both involving chromosomal rearrangements and changes in chromosome number) making $M$. persicae, as a whole, a complex, but intriguing, aphid species (Lauritzen 1982; Blackman 1987; Fenton et al. 1998; Spence and Blackman 


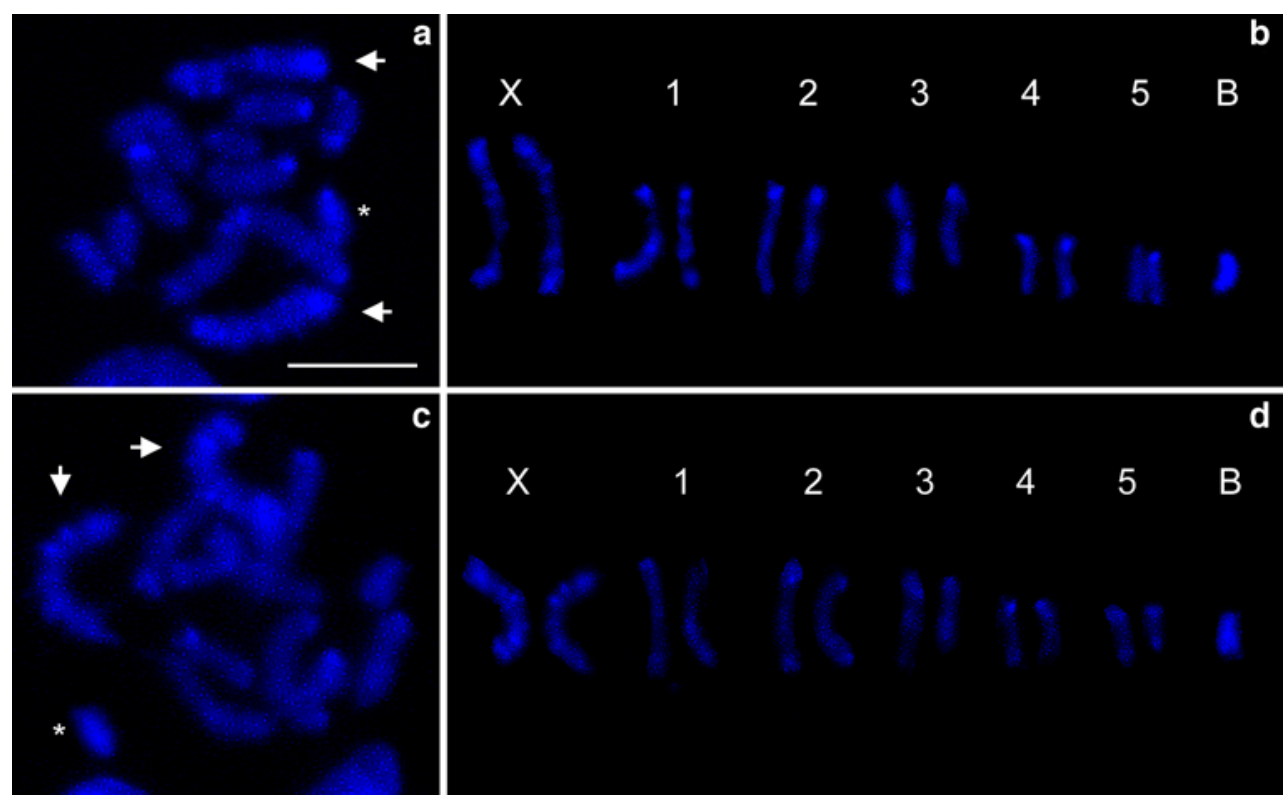

Fig. 5 DAPI staining of $\mathrm{C}$ banded chromosomes of the $M$. persicae clone $50(\mathbf{a}, \mathbf{b})$ and $51(\mathbf{c}, \mathbf{d})$ evidenced that the small supernumerary chromosome (indicated by the asterisk) is highly heterochromatic in both the clones as typical for B chromosomes. Arrows indicate the $\mathrm{X}$ chromosomes. Bar corresponds to $10 \mu \mathrm{m}$
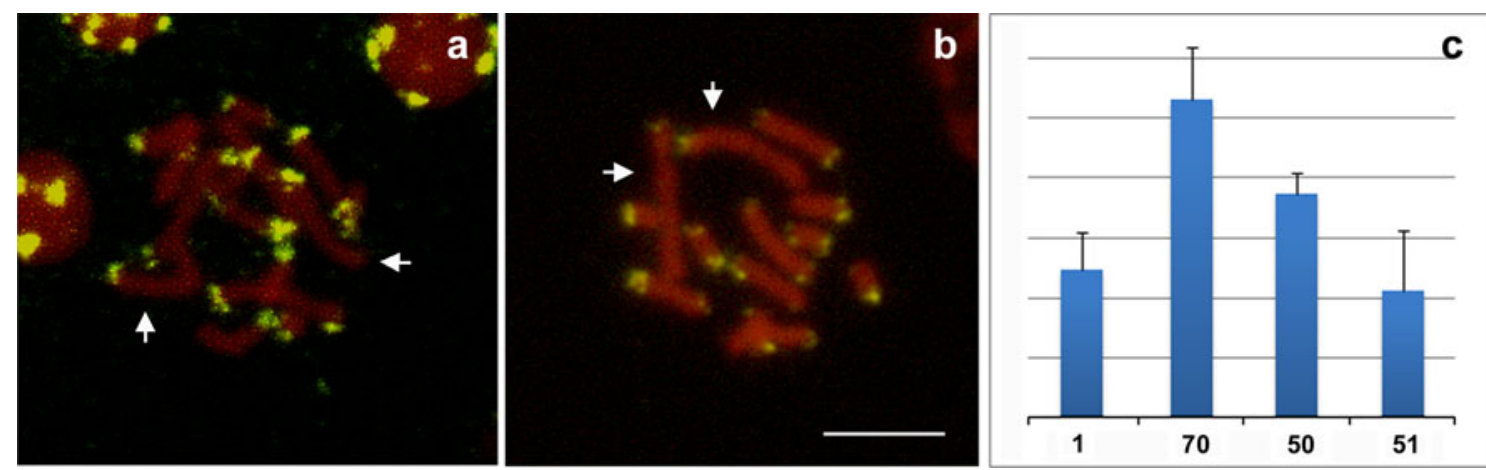

Fig. 6 FISH experiments with the FITC-labeled subtelomeric probe on propidium iodide-stained chromosomes evidenced a greater amplification level of subtelomeric repeat in clone 70 (a) when compared with clone 51 (b). Densitometric analysis of the dot blot experiments

1998; Loxdale 2007; Mandrioli et al. 1999b; Monti et al. 2012).

Analysis of mitotic metaphase chromosomes of M. persicae clones 50,51 and 70 revealed different chromosome numbers and karyotypes, in contrast to the standard karyotype of this species $(2 \mathrm{n}=12)$. In particular, we observed differences between embryos from different individuals within the same asexual lineage, between single embryos from the same individual, and also within each embryo. Chromosome length measurements, combined with FISH experiments, revealed that variant karyotypes were due to recurrent fragmentations of the chromosomes $\mathrm{X}, 1$ and 3, supporting earlier molecular studies reporting intraclonal genetic variations (Loxdale and Lushai 2003; with the subtelomeric probe confirmed that the amount of subtelomeric satellite DNA is greater in strains 50 and 70 than clones 1 and 51 (c). Arrows indicate X chromosomes. Bar corresponds to $10 \mu \mathrm{m}$

Lushai et al. 2003). These results can be explained by considering that the holokinetic structure of aphid chromosomes, combined with the apomictic mode of parthenogenesis, facilitates the inheritance of chromosomal rearrangements, since each fragment can be attached to microtubules so that they can be inherited without the constraint of homologous pairing typical of meiosis.

Our results confirm that autosomes 3 and, to a lesser extent 1 , are the chromosomes mostly involved in changes in the M. persicae karyotype (Spence and Blackman 1998; Monti et al. 2012) and support previous results also suggesting that the two $\mathrm{X}$ chromosomes can be fragmented or recombined (Monti et al. 2012). Furthermore, as observed after both FISH experiments with the subtelomeric repeat 

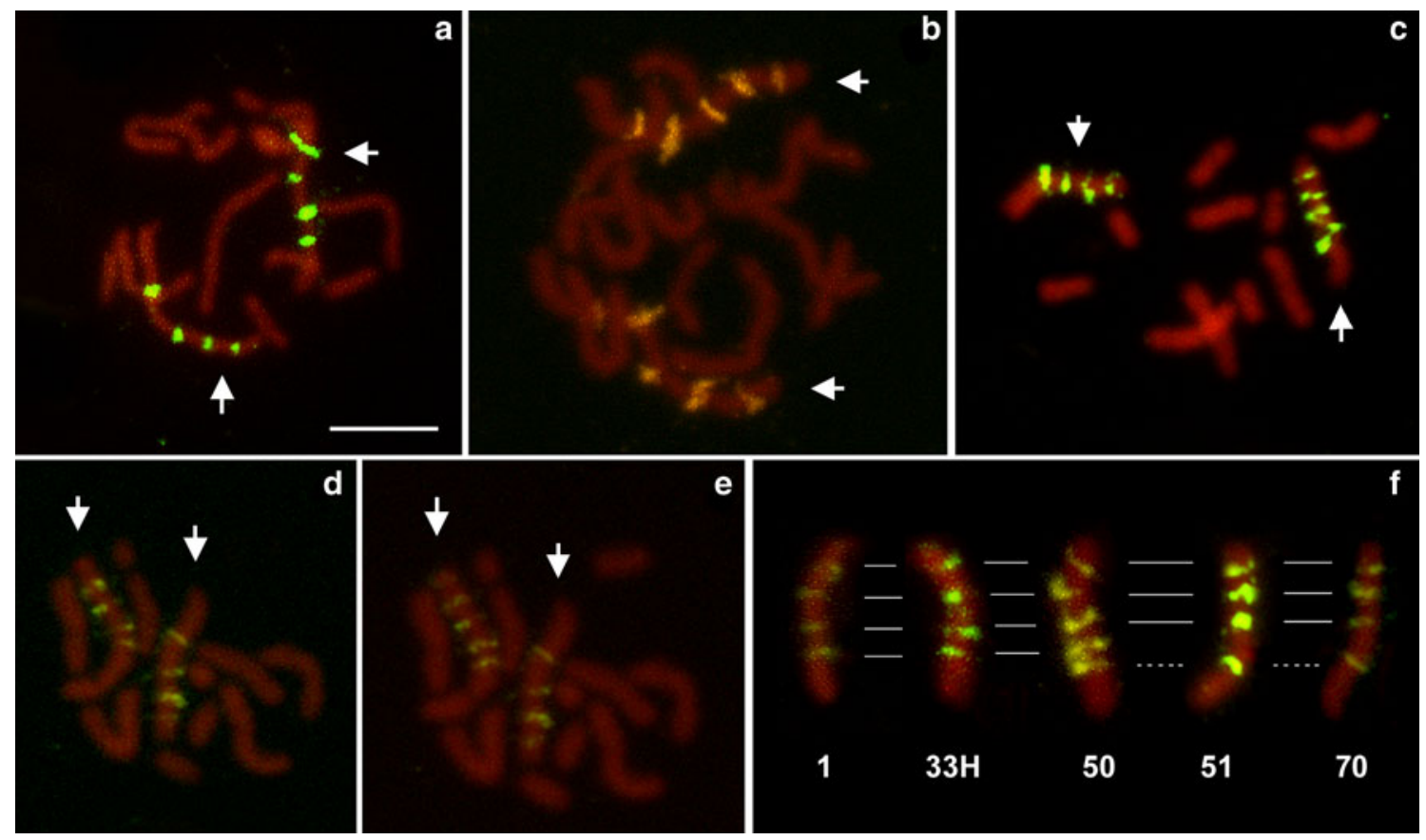

Fig. 7 FISH with the FITC-labelled Hind200 probe showed four hybridization bands on the $\mathrm{X}$ chromosomes of clones $33 \mathrm{H}$ (a), 51 (c), 70 (d) and 1 (e) and five bands in clone 50 (b), as evident in the

and Hind200 probes, $\mathrm{X}$ chromosomes bear duplications and inversions (as observed comparing the Hind200 hybridization pattern in clones 51 and 70) and differential amplification of heterochromatic regions (comparing the subtelomeric repeat hybridization pattern in clones 1,50 , 51 and 70).

The X chromosome fragmentation observed in $M$. persicae specimens of clone 70 occurred near a subtelomeric heterochromatic band enriched in satellite DNAs and this change is presumably unlikely to lead to any significant phenotypic change and therefore could perhaps be described as 'selectively neutral' (John 1983; Blackman et al. 2000). In contrast, the fragmentation of the $X$ chromosome observed in clone 51 involved euchromatic areas and may well have a phenotypic effect.

Up until a few years ago, fragmentations associated with the $\mathrm{X}$ chromosomes were considered rare in aphids not only in natural populations (Khuda-Bukhsh and Pal 1985; Hales 1989; Blackman et al. 2000), but also in X-ray irradiated aphids (Khuda-Bukhsh and Pal 1985). The previously reported stability of the $\mathrm{X}$ chromosomes was therefore only apparent and due to an inadequate coverage of aphid populations and/or species studied at a cytogenetic level, as also showed in aphids of the genus Trama (Blackman et al. 2000).

In accordance with previous results (Monti et al. 2012), the observed fragmentations of chromosomes 1 and 3 involved euchromatic areas and it would be interesting to comparison of the $\mathrm{X}$ hybridization pattern (f). Arrows indicate the $\mathrm{X}$ chromosomes. Bar corresponds to $10 \mu \mathrm{m}$

verify if they indeed affect some complex phenotypic traits, such as plant host choice. Indeed, several papers clearly indicate that chromosomal changes may affect host preference, as reported in Sitobion, Rhopalosiphum and Myzus aphids (Brown and Blackman 1988; De Barro et al. 1995; Hales et al. 2000), and may well have effects in aphid speciation events (Loxdale and Lushai 2007; Loxdale 2010b).

Several chromosomal plates in both clones 50 and 51 possessed a small and heterochromatic chromosome resembling a B chromosome. As reviewed in Camacho (2005), B chromosomes are additional dispensable chromosomes originated by A chromosomes showing a remarkably higher heterochromatin storage compared with A chromosomes. In view of the small length and large size of the subtelomeric repeat cluster at the telomeres, autosomes 3 and 4 are the most probable candidates for the origin of the observed B chromosome. Previous studies have identified supernumerary B chromosomes in aphids of the genus Euceraphis, derived from non-functional $\mathrm{X}$ chromosomes showing a greater within-species stability in size and number than typical B chromosomes (Blackman 1988). Our results therefore suggest for the first time that aphid B could originate also from autosomes.

$\mathrm{C}$ banding, followed by DAPI staining, showed that in both clones 50 and 51, B chromosomes are rapidly heterochromatinized, being highly enriched in heterochromatin. B chromosomes are generally enriched in satellite 
DNA and TEs and the accumulation of repeated DNA seems to be a very common event in B chromosomes differentiation. However, the occurrence of B chromosomes at an intra-clonal level in aphids argues that accumulation of repetitive DNA is not the primary cause of B chromosome differentiation. In particular, the rapid process of heterochromatinization could primarily result from epigenetic changes of the $\mathrm{B}$ chromosomes that are subsequently followed by structural modifications, including TE invasion and repetitive DNA amplification. Perhaps the chromosomal fragmentations presently seen, as suggested for intraclonal, intramorph RAPD (genomic) polymorphisms in aphids (e.g. Loxdale 2008a, b), may be due to TE effects, transposon rich 'hotspots' being known to cause rearrangements, i.e. inversion polymorphisms in other insects like Drosophila (Cáceres et al. 1999).

The idea that aphid populations are genetically stable on time and space is therefore erroneous since aphid clones are not genetically homogeneous as previously expected and a single female lineage does not represent a single evolutionary individual, as suggested by Janzen (1977), and earlier argued against on the basis of purely molecular biological data (Loxdale 2008a). On the contrary, as shown here also from chromosomal data and changes, aphid species seem to be the sum of populations that can have different karyotypes that in turn can give diverse genetic/ ecological/evolutionary responses in relation to imposed selective environmental forces.

Different studies suggest the presence of phenotypic plasticity in aphids (e.g. Wool and Hales 1997). However, in view of the presence of the large number of karyotypic and genetic changes observed in aphids (Loxdale 2008a, b; Monti et al. 2011, 2012) it is of interest to revisit these results in order to verify the nature of the observed plasticity in term of true plasticity (whatever this means exactly at a genetic level; see Whitman and Agrawal 2009) versus the sum of different non-plastic traits in different clones of the same species.

Experiments of male induction revealed that $M$. persicae clones 50, 51 and 70 are obligately parthenogenetic, suggesting that reproduction by apomictic parthenogenesis favours the stabilization and inheritance of the chromosomal fragments. Aphid populations bearing karyotype rearrangements reproduce generally by obligate parthenogenesis making their distribution limited to regions with warm winters (e.g. Delmotte et al. 2001). At present, climate warming can influence aphid distribution so that obligate parthenogenetic populations survive during winter making them more common now that in the past. Taking into account that chromosomal changes may well affect plant host choice (Brown and Blackman 1988; De Barro et al. 1995; Hales et al. 2000), it will be intriguing to follow the aforementioned $M$. persicae clones in the field.
This will involve verifying whether karyotype variants have effects over short temporal and spatial scales, as has been found to be the case with $M$. persicae strains with genetic/chromosomal variations related to insecticide resistance (e.g. Fenton et al. 2010).

Acknowledgments We are greatly indebted to Dr. Emanuele Mazzoni (Università Cattolica di Piacenza, Italy) for sending us the aphid strains. This work was supported by the grant "F.A.R." from the University of Modena and Reggio Emilia (M. M.) and by the grant "Experimental approach to the study of evolution" from the Department of Animal Biology of the University of Modena and Reggio Emilia (M. M.).

\section{References}

Blackman RL (1985) Aphid cytology and genetics. Evolution and biosystematics of aphids. In: Proceedings of the international symposium at Jablonna, Ossolineum, Wroclaw, pp 170-237

Blackman RL (1987) Morphological discrimination of a tobaccofeeding form from Myzus persicae (Sulzer) (Hemiptera: Aphididae), and a key to New World Myzus (Nectarosiphon) species. Bull Entomol Res 77:713-730

Blackman RL (1988) Stability of a multiple X chromosome system and associated B chromosomes in birch aphids (Euceraphis spp.; Homoptera: Aphididae). Chromosoma 96:318-324

Blackman RL, Spence JM, Normark BB (2000) High diversity of structurally heterozygous karyotypes and rDNA arrays in parthenogenetic aphids of the genus Trama (Aphididae: Lachninae). Heredity 12:254-260

Brown G, Blackman RL (1988) Karyotype variation in the corn leaf aphid, Rophalosiphon maidis (Fitch), species complex (Hemiptera, Aphididae) in relation to host plant and morphology. Bull Entomol Res 78:351-363

Cáceres M, Ranz JM, Barbadilla A, Long M, Ruiz A (1999) Generation of a widespread Drosophila inversion by a transposable element. Science 285:415-418

Camacho JPM (2005) B chromosomes. In: Gregory TR (ed) The evolution of the genome. Elsevier Academic Press, Burlington, pp 223-289

Crema R (1979) Egg viability and sex determination in Megoura viciae (Homoptera, Aphididae). Entomol Exp Appl 26:152156

De Barro PJ, Sherratt TN, David O, Maclean N (1995) An investigation of the differential performance of clones of the aphid S. avenae on two hosts. Oecologia 104:379-385

Delmotte F, Leterme N, Bonhomme J, Rispe C, Simon JC (2001) Multiple routes to asexuality in an aphid species. Proc R Soc Biol Sci B 268:2291-2299

Devonshire AL, Field LM, Foster SP, Moores GD, Williamson MS, Blackman RL (1999) The evolution of insecticide resistance in the peach-potato aphid, Myzus persicae. In: Denholm I, Pickett JA, Devonshire AL (eds) Insecticide resistance: from mechanisms to management. CABI Publishing, Wallingford, pp 1-9

Dixon AFG (1989) Parthenogenetic reproduction and the rate of increase in aphids. In: Minks A, Harrewijn P (eds) Aphids, their biology, natural enemies and control, vol A. Elsevier, The Netherlands, pp 269-287

Donlon TA, Magenis RE (1983) Methyl green is a substitute for distamycin $\mathrm{A}$ in the formation of distamycin A/DAPI C-bands. Hum Genet 65:144-146

Fenton B, Woodford JAT, Malloch G (1998) Analysis of clonal diversity of the peach-potato aphid, Myzus persicae (Sulzer), in 
Scotland, UK and evidence for the existence of a predominant clone. Mol Ecol 7:1475-1487

Fenton B, Margaritopoulos JT, Malloch G, Foster SP (2010) Microevolutionary change in relation to insecticide resistance in the peach-potato aphid, Myzus persicae. Ecol Entomol 35:131-146

Field LM, Blackman RL (2003) Insecticide resistance in the aphid Myzus persicae (Sulzer): chromosome location and epigenetic effects on esterase gene expression in clonal lineages. Biol J Linn Soc 79:107-113

Foster SP, Devine G, Devonshire AL (2007) Insecticide resistance. In: Van Emden HF, Harrington R (eds) Aphids as crop pests. CABI, UK, pp 261-286

Grimaldi D, Engel MS (2005) Evolution of the insects. Cambridge University Press, Cambridge

Hales D (1989) The chromosomes of Scoutedenia lutea (Homopteraa, Aphididae, Greenidinae) with an account of meiosis in the male. Chromosoma 98:295-300

Hales D, Wilson ACC, Spence JM, Blackman RL (2000) Confirmation that Myzus antirrhinii (Macchiati) occurs in Australia using morphometrics, microsatellite typing and analysis of novel karyotypes by fluorescent in situ hybridization. Aust J Entomol 39:123-129

Janzen DH (1977) What are dandelions and aphids? Am Nat 111:586-589

Jenkins RL (1991) Colour and symbionts of aphids. PhD thesis, University of East Anglia, UK

John B (1983) The role of chromosome change in the evolution of orthopteroid insects. In: Sharma AK, Sharma A (eds) Chromosomes in evolution of eukaryotic groups, vol I. CRC Press, Boca Raton, pp 1-110

Khuda-Bukhsh AR, Pal NB (1985) Cytogenetical studies on aphids (Homoptera: Aphididae) from India: I. Karyomorphology of eight species of Aphis. Entomologia 10:171-177

Lauritzen M (1982) Q- and G-band identification of two chromosomal rearrangements in the peach-potato aphids Myzus persicae (Sulzer), resistant to insecticides. Hereditas 97:95-102

Losey JE, Ives AR, Harmon J, Ballantyne F, Brown C (1997) A polymorphism maintained by opposite patterns of parasitism and predation. Nature 388:269-272

Loxdale HD (2007) Population genetic issues: the unfolding story revealed using molecular markers. In: Van Emden HF, Harrington R (eds) Aphids as crop pests. CABI Millennium Volume, CABI, UK, pp 31-67

Loxdale HD (2008a) Was Dan Janzen (1977) right about aphid clones being a 'super-organism', i.e. a single 'evolutionary individual'? New insights from the use of molecular marker systems. Mitt DGaaE 16:437-449

Loxdale HD (2008b) The nature and reality of the aphid clone: genetic variation, adaptation and evolution. Agric For Entomol 10:81-90

Loxdale HD (2009) What's in a clone: the rapid evolution of aphid asexual lineages in relation to geography, host plant adaptation and resistance to pesticides. In: Schon I, Martens K, van Dijk P (eds) Lost sex: the evolutionary biology of parthenogenesis. Springer, Heidelberg, pp 535-557

Loxdale HD (2010a) Setting the scene... meeting up with Darwin and Wallace. Ecol Entomol 35:1-9

Loxdale HD (2010b) Rapid genetic changes in natural insect populations. Ecol Entomol 35:155-164

Loxdale HD, Lushai G (2003) Rapid changes in clonal lines: the death of a 'sacred cow'. Biol J Linn Soc 79:3-16

Loxdale HD, Lushai G (2007) Population genetic issues: the unfolding story revealed using molecular markers. In: Van Emden HF, Harrington R (eds) Aphids as crop pests. CABI Millennium Volume, CABI, UK, pp 31-67
Lushai G, Loxdale HD, Brookes CP, von Mende N, Harrington R, Hardie J (1997) Genotypic variation among different phenotypes within aphid clones. Proc R Soc Lond B 264:725-730

Lushai G, De Barro PJ, Sherratt TN, Maclean N (1998) Genetic variation within a parthenogenetic lineage. Insect Mol Biol 7:337-344

Lushai G, Loxdale H, Allen JA (2003) The dynamic clonal genome and its adaptive potential. Biol J Linn Soc 79:193-208

Mandrioli M, Bizzaro D, Giusti M, Manicardi GC, Bianchi U (1999a) The role of rDNA genes in $\mathrm{X}$ chromosomes association in the aphid Acyrthosiphon pisum. Genome 42:381-386

Mandrioli M, Bizzaro D, Manicardi GC, Gionghi D, Bassoli L, Bianchi U (1999b) Cytogenetic and molecular characterization of a highly repeated DNA sequence in the peach potato aphid Myzus persicae. Chromosoma 108:436-442

Mandrioli M, Bizzaro D, Giusti M, Manicardi GC, Bianchi U (1999c) NOR heteromorphism within a parthenogenetic lineage of the aphid Megoura viciae. Chromosom Res 7:157-162

Mandrioli M, Azzoni P, Lombardo G, Manicardi GC (2011) Composition and epigenetic markers of heterochromatin in the aphid Aphis nerii (Hemiptera: Aphididae). Cytogenet Genome Res 133:67-77

Martens K, Loxdale HD, Schön I (2009) The elusive clone-in search of its true nature and identity. In: Schon I, Martens K, van Dijk P (eds) Lost sex: the evolutionary biology of parthenogenesis. Springer, Heidelberg, pp 187-200

Monti V, Giusti M, Bizzaro D, Manicardi GC, Mandrioli M, Rivi M

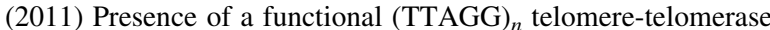
system in aphids. Chromosom Res 19:625-633

Monti V, Mandrioli M, Rivi M, Manicardi GC (2012) The vanishing clone: occurrence of repeated chromosome fragmentations in the aphid Myzus persicae (Homoptera, Aphididae). Biol J Linn Soc 105:350-358

Schweizer D (1976) Reverse fluorescent chromosome banding with chromomycin and DAPI. Chromosoma 58:307-324

Shufran KA, Mayo ZB, Crease TJ (2003) Genetic changes within an aphid clone: homogenization of rDNA intergenic spacers after insecticide selection. Biol J Linn Soc 79:101-105

Soumalainen E, Saura A, Lokki J (1987) Cytology and evolution in parthenogenesis. CRC Press, Boca Raton

Spence JM, Blackman RL (1998) Chromosomal rearrangements in the Myzus persicae group and their evolutionary significance. In: Nieto Nafria JM, Dixon AFG (eds) Aphids in natural and managed ecosystem. Universidad de Leon, Secretario de Publicaciones, Leon, pp 113-118

Spence JM, Blackman RL, Testa JM, Ready PD (1998) A 169 bp tandem repeat DNA marker for subtelomeric heterochromatin and chromosomal rearrangements in aphids of the Myzus persicae group. Chromosom Res 6:167-175

Sumner AT (1972) A simple technique for demonstrating centromeric heterochromatin. Exp Cell Res 75:304-306

Terradot L, Simon JC, Leterne N, Bourdin D, Wilson ACC, Guatier JP, Robert Y (1999) Molecular characterization of clones of the Myzus persicae complex differing in their ability to transmit the potato leafroll lutovirus (PLRV). Bull Entomol Res 89:255-263

Van Emden HF, Harrington R (2007) Aphids as crop pests. CABI, UK

van Toor RF, Fenton B, Malloch GL, Anderson EA, Dawson G (2012) Survival of sensitive and insecticide resistant genotypes of Myzus persicae on potato crops following application of different insecticide classes. Pest Manag Sci (in press)

Vorwerk S, Forneck A (2007) Analysis of genetic variation within clonal lineages of grape phylloxera (Daktulosphaira vitifoliae Fitch) using AFLP fingerprinting and DNA sequencing. Genome 50:660-667 
Whitman DW, Agrawal A (2009) What is phenotypic plasticity and why is it important? In: Whitman DW, Ananthakrishnan TN (eds) Phenotypic plasticity of insects: mechanisms and consequences. Science Publishers, Enfield, pp 1-63
Wool D, Hales DF (1997) Phenotypic plasticity in Australian cotton aphid: host plant effects on morphological variation. Ann Entomol Soc Am 90:316-328 